\title{
Nature-derived epigallocatechin gallate/duck's feet collagen/hydroxyapatite composite sponges for enhanced bone tissue regeneration
}

Yeon Ji Kook, Jingwen Tian, Yoo Shin Jeon, Min Jung Choi, Jeong Eun Song, Chan Hum Park, Rui L. Reis \& Gilson Khang

To cite this article: Yeon Ji Kook, Jingwen Tian, Yoo Shin Jeon, Min Jung Choi, Jeong Eun Song, Chan Hum Park, Rui L. Reis \& Gilson Khang (2017): Nature-derived epigallocatechin gallate/duck's feet collagen/hydroxyapatite composite sponges for enhanced bone tissue regeneration, Journal of Biomaterials Science, Polymer Edition, DOI: 10.1080/09205063.2017.1414480

To link to this article: https://doi.org/10.1080/09205063.2017.1414480

Dec 2017.

Published online: 20 Dec 2017.

Submit your article to this journal $₫$

Џ Article views: 7

Q View related articles ¿ᄌ

View Crossmark data \ulcorner 


\title{
Nature-derived epigallocatechin gallate/duck's feet collagen/ hydroxyapatite composite sponges for enhanced bone tissue regeneration
}

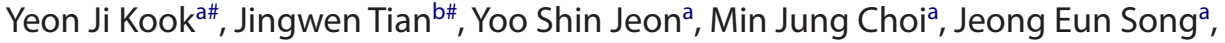 \\ Chan Hum Parkc, Rui L. Reis ${ }^{d}$ and Gilson Khang ${ }^{a}$ \\ aDepartment of BIN Convergence Technology, Department of Polymer Nano Science \& Technology and Polymer

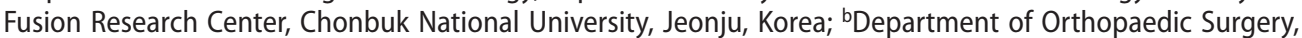 \\ Medical School and Research Institute of Clinical Medicine, Chonbuk National University, Jeonju, Republic \\ of Korea; 'Nano-Bio Regenerative Medical Institute, School of Medicine, Hallym University, Chuncheon, \\ Republic of Korea; '3B's Research Group - Biomaterials, Biodegradables and Biomimetics, University of Minho, \\ Headquarters of the European Institute of Excellence on Tissue Engineering and Regenerative Medicine, \\ Guimaraes, Portugal
}

\begin{abstract}
Scaffolds mimicking structural and chemical characteristics of the native bone tissues are critical for bone tissue engineering. Herein, we have developed and characterized epigallocatechin gallate/ duck's feet collagen/hydroxyapatite (EGCG/DC/HAp) composite sponges that enhanced the bone tissue regeneration. The threedimensional composite sponges were synthesized by loading various amounts (i.e. 1, 5 and $10 \mu \mathrm{M}$ ) of EGCG to duck feet derived collagen followed by freeze-drying and then coating with hydroxyapatite. Several measuremental techniques were employed to examine the properties of the as-fabricated composite sponges including morphology and structure, porosity, compressive strength, etc. and as well compared with pristine duck feet derived collagen. SEM observations of EGCG/DC/HAp sponges showed the formation of a highly porous collagen matrix with EGCG embodiment. The porosity and pore size of sponges were found to increase by high EGCG content. The compressive strength was calculated as $3.54 \pm 0.04$, $3.63 \pm 0.03,3.89 \pm 0.05,4.047 \pm 0.05 \mathrm{MPa}$ for 1,5 and $10 \mu \mathrm{M} \mathrm{EGCG/DC/}$ HAp sponges, respectively. Osteoblast-like cell (BMSCs isolated from rabbit) culture and in vivo experiments with EGCG/DC/HAp sponges implanted in nude mouse followed by histological staining showed enhanced cell internalization and attachment, cell proliferation, alkaline phosphatase expressions, indicating that EGCG/DC/HAp sponges have ahigh biocompatibility. Moreover, highEGCG content in the EGCG/DC/HAp sponges have led to increased cellular behavior. Collectively, the $5 \mu \mathrm{M}$ of EGCG/DC/HAp sponges were suggested as the potential candidates for bone tissue regeneration.
\end{abstract}

\section{ARTICLE HISTORY}

Received 11 April 2017

Accepted 23 November 2017

\section{KEYWORDS}

Duck's feet collagen (DC); epigallocatechin gallate (EGCG); hydroxyapatite (HAp); bone regeneration; bone marrow stromal cells (BMSCs)

CONTACT Gilson Khang gskhang@chonbuk.ac.kr

"Both authors contributed equally. 


\section{Introduction}

Bone tissue engineering plays an important role in treating human bone defect mostly caused by traffic accidents, innate disease or acquired disease [1]. Scaffolds portray a crucial role in bone tissue engineering because of their efficient capability of mimicking bone extracellular matrix (ECM) functions. Native bone ECM is a 3D porous and organic-inorganic composite materials that supporting cellular adhesion, differentiation and further growth. The organic phase includes collagen and non-collagenous proteins, sulfated and non-sulfated glycosaminoglycans, whereas the mineral phase of bone comprises semi-crystalline carbonated hydroxyapatite capable of standing against compressive load [2]. Hence, the selection of ideal biomaterial for tissue-engineered constructs are crucial to guide cells into functional tissue via improving cell adhesion, proliferation and differentiation.

We choose a naturally derived polymer, duck's feet collagen with good cell seeding and cell attachment and rapid biodegradation $[3,4]$. The fabrication method and biocompatibility of duck's feet collagen were already evaluated in our previous study [5,6]. Moreover, DC has been widely used as a base material for tissue-engineered bone constructs previously, as well many studies reported the incorporation of various osteo-inductive elements i.e. bone growth factors and cytokines [7-9]. However, studies regarding to the usage of plant/ herbal extracts for bone regeneration are scanty. Approximately 25\% of available drugs which are allowed to prescript were derived from plants, trees, and herbs [10]. Inspired by those medicinal values, we have employed epigallocatechin gallate (EGCG) which is the most abundant catechin found in green tea, onion and apple skin, contributing to various beneficial health effects associated with their consumption [11-14]. EGCG, known as epigallocatechin-3-gallate, is an ester of epigallocatechin and gallic acid, and is a type of catechin [15]. Previous reports have shown that EGCG in combination with $\alpha$-tricalcium phosphate particles can stimulate bone regeneration by increasing the formation of mineralized bone nodules with human osteoblast-like cells [16]. We hypothesize that EGCG usage, as a nature derived biomaterial for bone tissue regeneration instead of growth factors and cytokines, not only reduce scaffold's cost and risk, but also provide medicinal benefits.

MSC exist in almost all tissues including bone marrow, adipose tissue, cartilage. They have the ability to migrate into sites of injury and differentiated toward terminally committed cells for use in regenerative medicine. Bone marrow stromal stem cells (BMSCs) possess the capability of self-renewed and differentiation into organ-specific cell types. Due to excellent proliferation and immunologic characteristics BMSCs appear to be ideal seeding cells. The cell population harvested from bone marrow cells in various conditioned media can make specific cell phenotype and amounts available for graft construction.

The main aim of our study is to design an efficient bone graft that promotes bone growth in the bone defect region via inducing BMSCs proliferation and differentiation. In this study, we have fabricated epigallocatechin gallate/duck's feet collagen/hydroxyapatite (EGCG/ DC/HAp) composite sponges as a function of EGCG contents (i.e. 1, 5 and $10 \mu \mathrm{M}$ ) via freeze-drying for enhanced bone tissue regeneration. The efficiency of the EGCG/DC/HAp composite sponges were evaluated for osteogenic differentiation of BMSCs in both in vitro and in vivo environments. Physiological, chemical, thermal and mechanical characteristics of the sponges were examined using scanning electron microscopy and Fourier transform infrared spectroscopy (FTIR). 


\section{Materials and methods}

\subsection{Materials}

For this study, Raw Duck's feet were purchased from Korean local market. EGCG, sodium hydroxide, chloroform, acetone, methanol, citric acid, pepsin, and acetic acid were obtained from Sigma-Aldrich. All reagents used in this experiment were high-performance liquid chromatography (HPLC) grade.

\subsection{Preparation of duck's feet collagen}

Duck's feet collagen (DC) was prepared according to our previously reported study [17]. In brief, iced duck's feet was washed with distilled water and immersed in $0.5 \mathrm{M}$ sodium hydroxide solution for $24 \mathrm{~h}$ to remove fat from tissue followed by washing with methanol: chloroform solution in 3:1 ratio, acetone, alcohol and distilled water, respectively. For collagen extraction, the feet were again submerged in 5\% citric acid for $24 \mathrm{~h}$ and then mixed with $3 \mathrm{~g}$ pepsin for $48 \mathrm{~h}$. Then the supernatant was collected. To obtain precipitated DC, the supernatant was centrifuged at $10,000 \mathrm{rpm}$ for $10 \mathrm{~min}$, filtered with gauze and centrifuged at $3500 \mathrm{rpm}$ with ethanol. Finally, the obtained products were lyophilized. Prior to the fabrication of composite sponges, the lyophilized DC was added to $0.5 \mathrm{M}$ acetic acid and stirred for 3 days (2\% DC solution).

\subsection{Fabrication of EGCG/DC/HAp sponges}

The EGCG/DC sponges were prepared using the freeze-drying method. First, $10 \mu \mathrm{M}$ EGCG was prepared in distilled water under stirring at $60 \mathrm{rpm}$ for $30 \mathrm{~min}$. Different concentrationof $(1,5,10 \mu \mathrm{M})$ EGCG solution poured into $2 \%$ collagen solution in exact quantity. The collagen solution loaded EGCG was then poured into 48 well plate $(1 \mathrm{~mL} /$ well $)$ and frozenat $-80^{\circ} \mathrm{C}$, thawed at room temperature at an interval of $6 \mathrm{~h}$ for three times. Next, the EGCG/DC/HAp sponges were prepared according to the following method: dried $2 \%$ EGCG/DC sponges were immersed in 1.0X stimulated body fluid (SBF) solution for $24 \mathrm{~h}$, washed in distilled water and lyophilized for $48 \mathrm{~h}$.

\subsection{Sponges characterizations}

The sponges' morphology were examined by SEM. The chemical properties of sponges were analyzed by Fourier transform infrared spectroscopy (FTIR, Spectrum GX, Perkin Elmer, USA) in the range of 4000-500 $\mathrm{cm}^{-1}$. The compressive mechanical strength and height of the sponges were measured by TMS-Pro instrument (Food Technology Corporation, Sterling, VA, USA) where the samples were moved down at a target distance to specimen of $1.5 \mathrm{~mm}$ with a speed of $10 \mathrm{~mm} / \mathrm{s}$ and force of $0.5 \mathrm{~N}$.

\subsection{Isolation and culture of bone marrow derived mesenchymal stem cells}

BMSCs were isolated from New Zealand White rabbits (6 weeks old, female). Briefly, bone marrow was harvested from the tibia and femur condyle. BMSCs were isolated from bone marrow by ficoll density-gradient centrifugation. Then, the BMSCs were plated in 
alpha-minimum essential culture medium ( $\alpha$-MEM) (Lonza, Walkersville, MD, USA) containing $20 \%$ fetal bovine serum, and $1 \%$ penicillin/streptomycin (Invitrogen, Carlsbad, CA, USA) at $37{ }^{\circ} \mathrm{C}$ and $5 \% \mathrm{CO}_{2}$. The culture medium was replaced in every third day. BMSCs were seeded into each sponge at a concentration of $1 \times 10^{5}$ cells.

\subsection{In vitro cell proliferation}

Prior to cell seeding experiments, the sponges were sterilized with $70 \%$ ethanol about $30 \mathrm{~min}$, removed ethanol with phosphate buffer saline (PBS) about $20 \mathrm{~min}$ and hydrated with $a-M E M$ in the incubator. Then, passage 1 BMSCs were detached from the dishes and seeded into the sponges at a density of $1 \times 10^{5}$ /sponges. After culturing of the cells on EGCG/ DC/HAp sponges for 7 and 28 days, the sponges were washed with PBS and fixed with $2.5 \%$ SEM-grade glutaraldehyde (diluted in $1 \mathrm{X}$ PBS) for $24 \mathrm{~h}$ at room temperature. The samples were dehydrated using series of ethanol solutions (increasing from 50 to 100\%). Finally, the images were observed by SEM (SN-SUPRA 40VP, Carl Zeiss, Germany).

The cell proliferation on the sponges were measured using MTT (3-4-2, 5-diphenyl tetrazolium bromide, Sigma-Aldrich, USA) assay. In brief, BMSCs were seeded on the as-prepared sponges for 1, 7, 14 and 21 days. One hundred milliliter of MTT solution was added to the sponges and incubated for $4 \mathrm{~h}$ at $37^{\circ} \mathrm{C}$ and $5 \% \mathrm{CO}_{2}$. After the formation of purple crystals, the solution was removed and sponges were carefully washed with PBS. One microliter of dimethylsulfoxide (DMSO, Sigma-Aldrich) was added and incubated for $24 \mathrm{~h}$. One hundred milliliter of the solution was pipetted into 96-well plate, and the absorbance was noted at $570 \mathrm{~nm}$ using Synergy Mx monochromator-based multi-mode microplate reader (Biotek instruments, Inc., USA).

\subsection{Alkaline phosphatase activity}

The osteogenic differentiation of BMSCs on sponges were measured with ALP Assay Kit (Takara Bio Inc., Tokyo, Japan) according to the manufacture's protocol. BMSCs were seeded in the sponges at the concentration of $5 \times 10^{4} /$ sponge and cultured in culture medium for 1 , 7, 14 and 21 days. At each time point, the samples were rinsed with PBS. The protein from each BMSCs seeded with sponge extracted using 5\% alkaline phosphatase (ALP) extraction solution, and added para-nitrophenyl phosphate ( $\mathrm{pNPP}$ ) solution. The reaction solution incubated at $37^{\circ} \mathrm{C}$ and $5 \% \mathrm{CO}_{2}$ for $1 \mathrm{~h}$. The reaction was stopped by adding $0.9 \mathrm{~N} \mathrm{NaOH}$ stop solution. The absorbance was measured at $405 \mathrm{~nm}$ using microplate reader (Biotek instruments, Inc., USA).

\subsection{Real-time polymerase chain reaction}

Further, osteogenic gene expression was evaluated by reverse transcription polymerase chain reaction (RT-PCR). After 1, 7, 14 and 21 days of cell culture in sponges, total RNA was extracted using a Trizol reagent (Takara Bio Inc., Tokyo, Japan) and $0.2 \mathrm{~mL}$ chloroform. The supernatants precipitated with $0.5 \mathrm{~mL}$ of isopropanol (Sigma-Aldrich) and $5 \mu \mathrm{L}$ of Polyacryl Carrier (Molecules Research Center, Inc., Cincinnati, OH, USA). The RNA samples were reverse transcribed into cDNA using Oligo (dT) primer (Invitrogen ${ }^{\mathrm{Tm}}$ ), each of primers; Glyceraldehyde 3-phosphate dehydrogenase (GAPDH), type-I collagen (Col-I), 
and osteocalcin (OCN). We observed that all PCR products were separated by electrophoresis on $1.2 \%(\mathrm{w} / \mathrm{v})$ agarose gel containing ethidium bromide $(\mathrm{EtBr})$ which was visualized under UV light (FluorChem HD2 Gel Imaging System, Alpha Innotech) at $300 \mathrm{~nm}$. The primers used in this study were purchased from Genotec (Daejon, Korea).

\subsection{Histological evaluation}

Histological evaluation of bone tissue differentiation was performed after 2 and 4 weeks post-surgery. For implantation, we sterilized sponges. In the clean bench, the sponge was placed in a 24 well plate according to each experiment group $(n=3)$. To sterilize sponges, $1 \mathrm{ml}$ of $70 \%$ alcohol was placed in a well plate containing sponge for $30 \mathrm{~min}$ under UV lamp. Then, remove the 70\% alcohol and rinsed it 2 times in PBS solution for $20 \mathrm{~min}$. Lastly, PBS was removed and alpha mem medium was placed in a well plate containing sponges then incubation before BMSCs seeding.

BMSCs seeded sponges were implanted into the subcutaneous armpit of four weeks old athymic nude mouse (100-150 g, female, Hanil laboratory animal center, Korea). Nude mice were anesthetized with intramuscular injection with $150 \mu \mathrm{L}$ of Zoletil (Virbac S. A, FRA) and Domitor (Orion Pharma, FIN) by ratio of 2:1. The surgical site was washed with povidone-iodone. Cell-sponge constructs were implanted into the subcutaneous dorsum of nude mouse ( $n=2$ per each group, 20 mice). Then the implants were harvested after 2 and 4 weeks. All experiment procedures were performed with the approval of the Chonbuk National University Animal Care Committee, Jeonju, Korea (CBNU 2016-50).

Subsequently, the collected implants were fixed in 10\% formaldehyde (Sigma-Aldrich), dehydrated with a series of graded ethanol, and specimens were embedded in paraffin. Paraffin sections of $8-\mu \mathrm{m}$ thickness were conducted by microtome (Thermo Scientific) and fixed on poly-L-lysine (PLL) coating slide. Sections were stained with hematoxylin and eosin ( $\mathrm{H} \& \mathrm{E})$ and von kossa after the de-paraffin process for histological examination. All the samples were analyzed under an optical microscope.

\subsection{Statistical analysis}

The data were expressed as means \pm Standard Deviation (SD) of experiments performed in triplicate. Statistical significance was analyzed using Student's $t$-test and $p$ values less than 0.05 is accepted to indicate statistically significant differences.

\section{Result and discussion}

\subsection{Morphology of EGCG/DC/HAp sponges}

Figure 1 showed the (a) gross and (b) cross-sectional images of DC/HAp, and 1, 5 and $10 \mu \mathrm{M}$ EGCG/DC/HAp composite sponges. All the sponges presented a similar elliptical shape and a smooth, soft and sponge-like morphology along with thickness of $2 \mathrm{~mm}$ and wide length of $\sim 7 \mathrm{~mm}$. These uniform elliptical shapes can be ascribed to the $3 \mathrm{~h}$ refrigeration, freeze- drying and lyophilization [18]. 
(a)

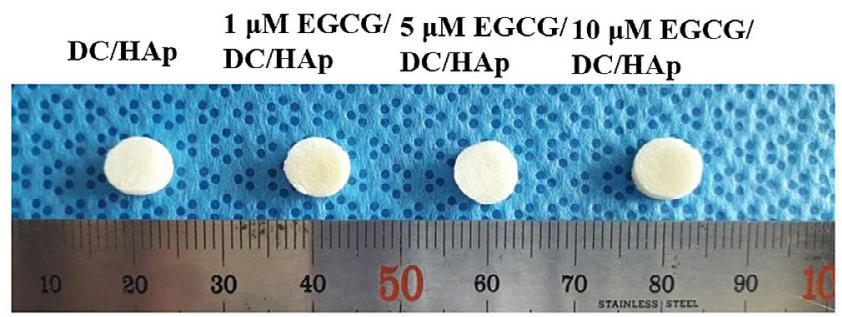

(b)
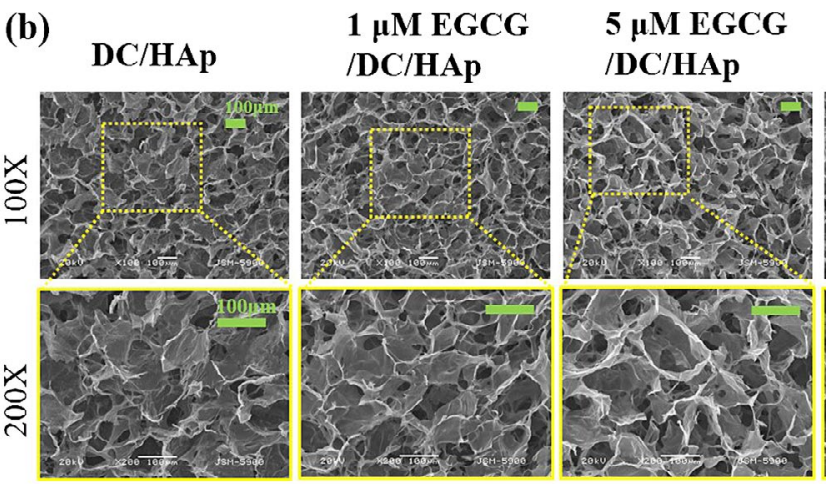

$10 \mu \mathrm{M}$ EGCG $/ \mathbf{D C} / \mathbf{H A p}$

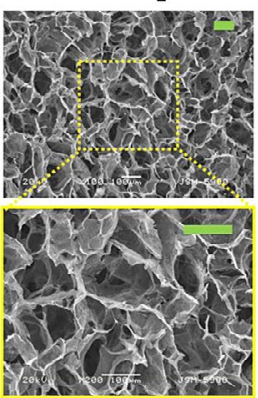

Figure 1. (a) Gross images, and (b) cross-section SEM image of as-fabricated 1, 5 and $10 \mu \mathrm{M} \mathrm{EGCG/DC/}$ HAp sponges.

\subsection{Mechanical properties}

Figure 2 showed the (a) FTIR spectra, (b) compressive strength, and (c) porosity of bare DC/HAp and 1, 5 and $10 \mu \mathrm{M}$ EGCG/DC/HAp sponges. The FTIR spectrum (Figure 2(a)) of $2 \%$ DC displayed several characteristic peaks at 1780 and $900 \mathrm{~cm}^{-1}$, ascribed to amide-I and amide-II. The hydroxyapatite spectrum showed peaks at 1114 and $1002 \mathrm{~cm}^{-1}$, assigned to $\mathrm{P}-\mathrm{O}$ phosphate groups stretching of three, and peaks at 968 and $943 \mathrm{~cm}^{-1}$ were attributed to $\mathrm{P}-\mathrm{O}$ bonds banding modes in the phosphate groups. Moreover, the FTIR spectra of HAp showed well-preserved characteristic peaks within the DC/HAp sponges [19], thus suggesting that no significant structural changes of DC/HAp throughout the composite formation. Further EGCG/DC/HAp sponges' spectrum showed all DC/HAp and EGCG defined peaks, which further intensified with an increase in EGCG contents. According to the porosity results (Figure 2(c)), DC/HAp, $1 \mu \mathrm{M}$ EGCG/DC/HAp, $5 \mu \mathrm{M}$ EGCG/DC/HAp sponges showed $52.27 \pm 3.12,47.35 \pm 5.53,55.02 \pm 5.07,60.42 \pm 2.94 \%$, respectively. The mechanical properties of the sponges were studied by measuring compression strength of sponges (Figure 2(b)). The samples showed $3.54 \pm 0.04,3.63 \pm 0.03,3.89 \pm 0.05,4.047 \pm 0.05 \mathrm{MPa}$ for DC/HAp and EGCG/DC/HAp sponges. The addition of EGCG was found to improve the compressive strength of the EGCG/DC/HAp sponges as compared to DC/HAp sponge.

\subsection{Osteogenic differentiation of BMSCs on DC/HAp and EGCG/DC/HAp sponges}

The osteogenic differentiation of BMSCs on DC/HAp and EGCG/DC/HAp composite sponges were evaluated by culturing BMSCs on the sponges and examined by SEM observation, MTT assay and ALP assay. The SEM images (Figure 3) displayed the interconnected 


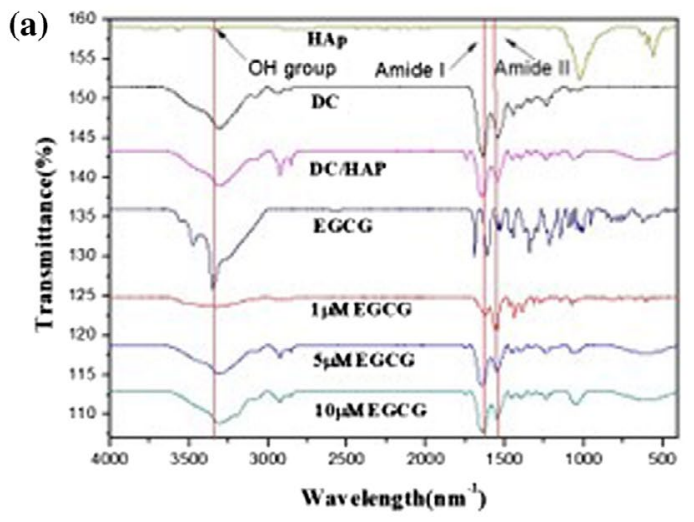

(b)

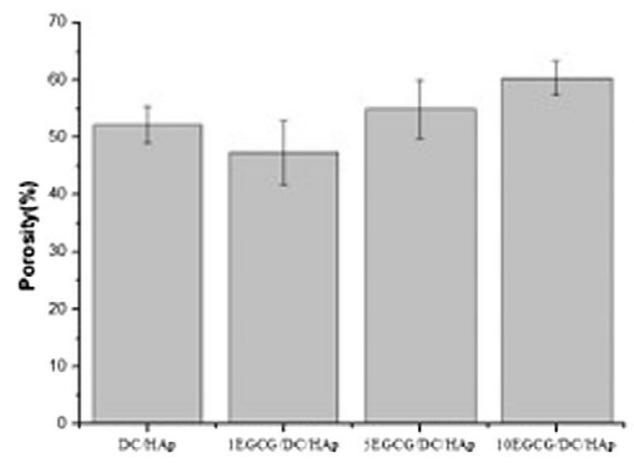

(c)

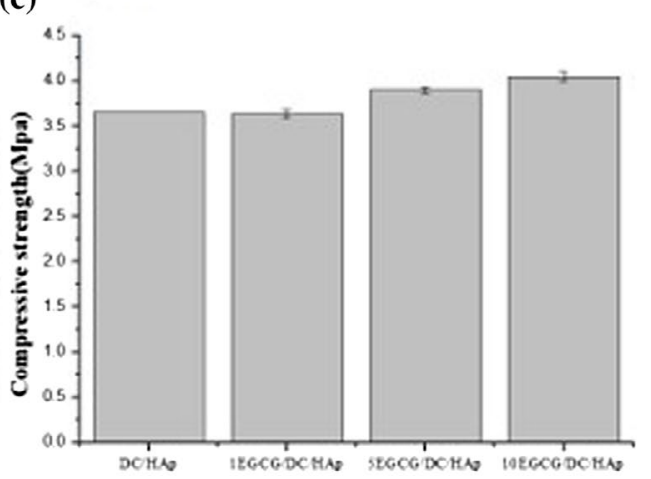

Figure 2. (a) FTIR spectra, (b) Porosity, and (c) Compressive strength of 1, 5 and $10 \mu \mathrm{M} \mathrm{EGCG/DC/HAp}$ sponges $(n=3)$.

pores sponges. The interconnected pores are critical for transporting essential nutrients, oxygen to cells and removal of cell debris. The architecture of micro-periodic construct has been reported to enhance the bone regeneration in vivo [20]. The morphology of cultured BMSCs on EGCG/DC/HAp composite sponges was noticed after 7 and 28 days. The morphology of BMSCs on both DC/HAp and EGCG/DC/HAp sponges showed round shape and osteoblastic-like morphology in the pores surrounded by ECM on 7th day of culture. Compared to the EGCG/DC/HAp sponges, DC/HAp sponges displayed a very few cells. Interestingly, significantly high number of cells were especially surrounded in the $5 \mu \mathrm{M}$ EGCG/DC/HAp sponges on day 28, thus these results suggesting that the EGCG compound efficiency for supporting cell proliferation and attachment.

Figure 4 showed the osteogenic differentiation of BMSCs on cultured on 1,5 and $10 \mu \mathrm{M}$ EGCG/DC/HAp sponges for 1, 7, 14, 21 and 28 days studied by MTT assay and ALP assay. The MTT assay (Figure 4(a)) was performed at 1, 7, 14, and 21 days of BMSCs culturing to evaluate the cell proliferation on DC/HAp and EGCG/DC/HAp sponges. Until 7 days of cell culture, no significant difference was observed between DC/HAp and EGCG/DC/HAp sponges. However, on 14th and 21st day, the $5 \mu \mathrm{M}$ of EGCG/DC/HAp sponge showed higher cell proliferation rate than the bare DC/HAp and 1, $10 \mu \mathrm{M}$ EGCG/DC/HAp sponges. This result indicates that the potency of EGCG content present in EGCG/DC/HAp sponges for promoting cell attachment and proliferation. Furthermore, the osteogenic differentiation of 


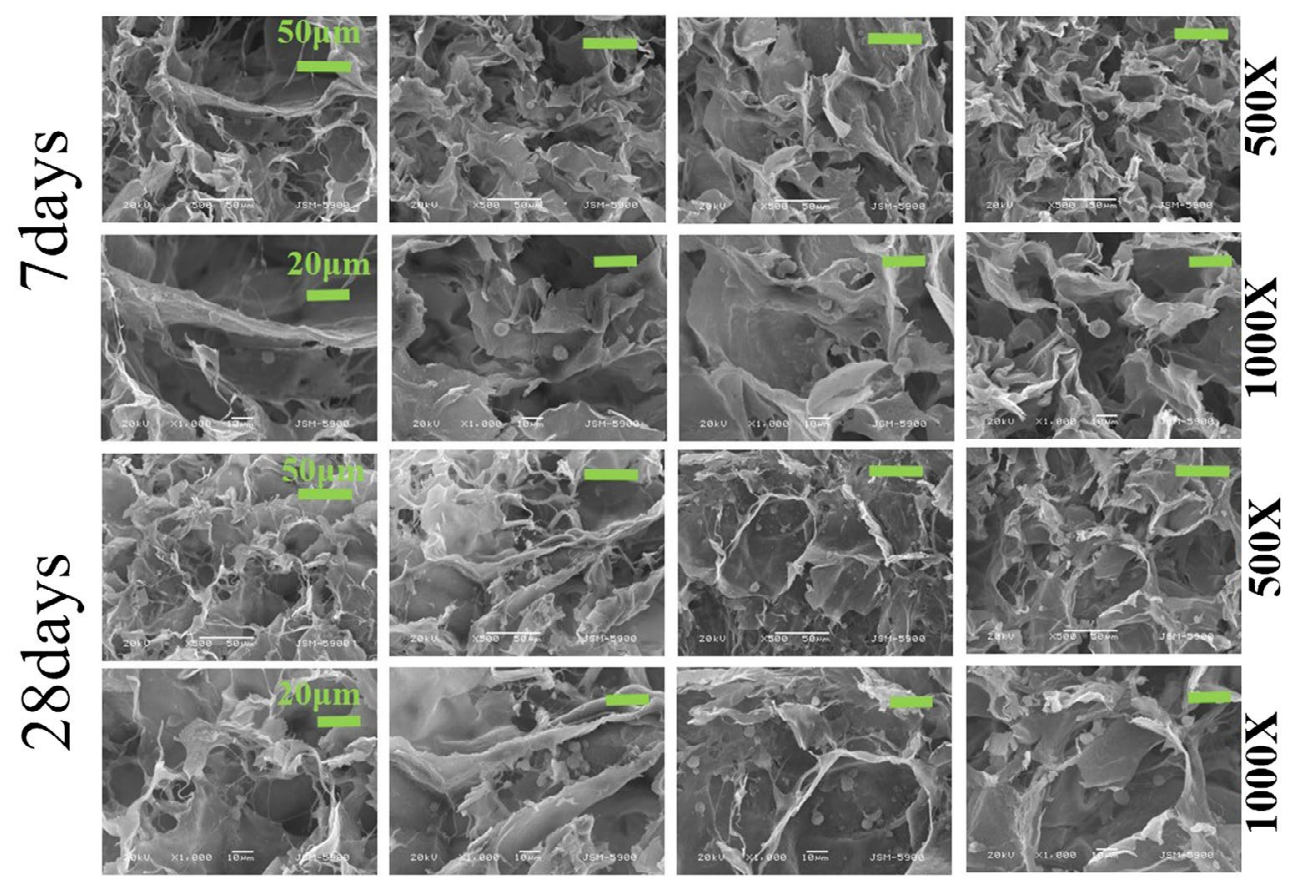

Figure 3. SEM images of BMSCs cultured on the as-fabricated 1, 5 and $10 \mu M$ EGCG/DC/HAp sponges for 21 days, showing enhanced cell attachment and proliferation $(n=3)$.

(a)

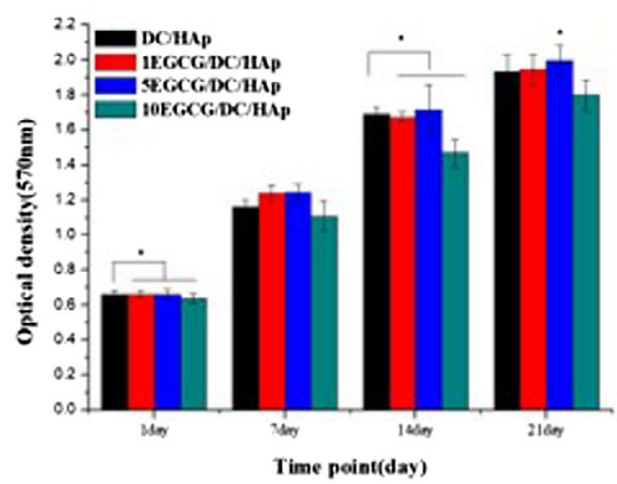

(b)

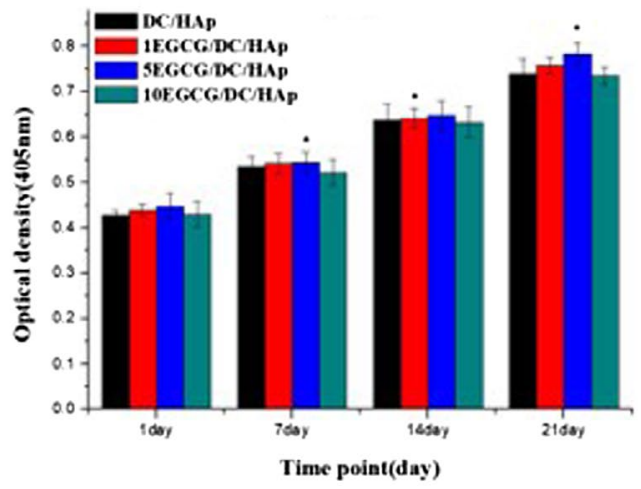

Figure 4. Osteogenic differentiation of BMSCs on cultured on 1, 5 and $10 \mu M$ EGCG/DC/HAp sponges for $1,7,14,21$ and 21 days studied by (a) MTT assay and (b) ALP assay ( $n=3$ in each group, $p^{*}<0.05$ ).

BMSCs on 1, 5 and $10 \mu \mathrm{M}$ EGCG/DC/HAp sponges were also confirmed by ALP assay, as shown in Figure 4(b). At 1st and 7th day of cell culture, the ALP activity of BMSCs showed no significant difference between bare DC/HAp and EGCG/DC/HAp sponges. Presenting a consistency with the MTT assay, the cultured BMSCs on $5 \mu \mathrm{M}$ EGCG/DC/HAp sponges demonstrated higher ALP activity on 14th and 21st day. 


\subsection{RT-PCR analysis}

To study the ability of osteogenic differentiation of BMSCs on DC/HAp and EGCG/DC/HAp sponges, qRT-PCR was performed to detect the expressions of bone-specific genes such as OCN, RUNX-2 and COL1 after 21 days cell culture and GAPDH was used to normalized the data as shown in Figure 5(a) and (b). OCN plays an important role in pro-osteoblast and bone formation. RUNX-2 gene was associated with osteoblast differentiation [21]. COL1 also plays an important role in producing osteoblast and depositing mineral [22]. Compared with the gene expression on bare DC/HAp sponges, gene expression was showed upregulated evidently for EGCG/DC/HAp sponges. Notably, real-time quantitative (RQ) values of OCN (1.47994), RUNX-2 (1.8794) and COL1 (0.88362) on the $5 \mu \mathrm{M}$ EGCG/DC/ HAp sponges displayed highest expressions. Its suitability for further in vivo studies was confirmed by the in vitro studies indicated the favorable effects of EGCG on BMSCs osteogenic differentiation and ECM mineralization. Thus biological evaluation qualifies of the $5 \mu \mathrm{M}$ EGCG/DC/HAp sponges as a potential construct for bone tissue regeneration. EGCG at concentrations of 1 and $5 \mu \mathrm{M}$ caused a dose-dependent increase expression as assessed by gel image. But $10 \mu \mathrm{M}$ is decreased expression compared other experiment groups. This because excessive addition of EGCG suppressed osteoblast maturation by inhibiting latestage differentiation [23]. It may partially contribute to the supressive effects of EGCG on osteodifferentiation.

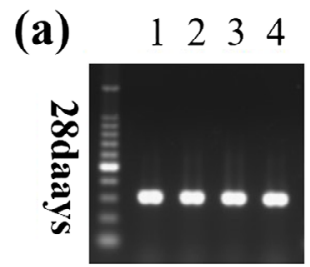

GAPDH

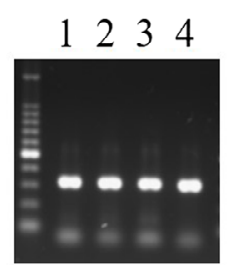

OCN

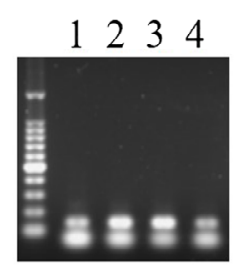

RUNX2

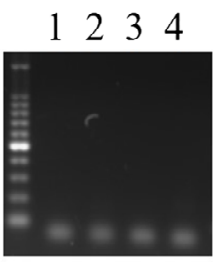

COL1

1. DC/HAp 2. 1EGCG/DC/HAp 2.5EGCG/DC/HAp 4. 10EGCG/DC/HAp

(b)

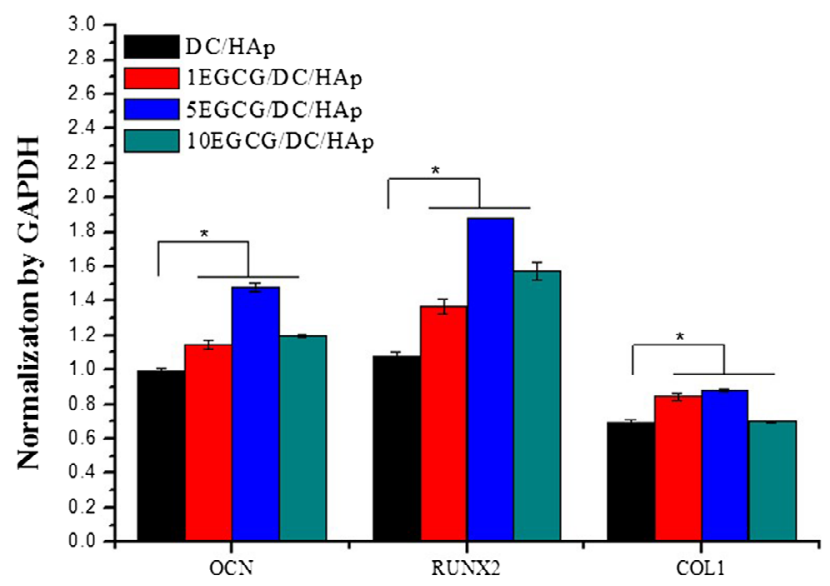

Figure 5. mRNA expression of BMSCs analyzed by RT-PCR on 21 days. (a) Electrophoresis assay using specific cell markers. (b) Normalization by GAPDH $\left(n=3\right.$ in each group, $\left.p^{*}<0.05\right)$. 


\subsection{Histological analysis}

The in vivo osteoinduction and osteoconduction ability of the as-fabricated DC/HAp and EGCG/DC/HAp sponges were examined by implanting the sponges on a full thickness the subcutaneous region of athymic nude mice followed by their sacrifice at 2 and 4 weeks post-surgery for staining experiments. Figure 6 showed the histological evaluation of EGCG/ DC/HAp sponges implanted under the subcutaneous region of athymic nude mice for 2 and 4 weeks using hematoxylin and eosin $(\mathrm{H} \& \mathrm{E})$. Compared to the DC/HAp sponges, $\mathrm{H} \& \mathrm{E}$ staining results displayed denser stains and thicker bone matrix for the EGCG/DC/HAp sponges. Moreover, the $5 \mu \mathrm{M}$ EGCG/DC/HAp sponges showed well-defined construct, higher osteoblast infiltration and distribution among the EGCG/DC/HAp sponges. Figure 7(a) showed the histological evaluation of EGCG/DC/HAp sponges implanted under the subcutaneous region of athymic nude mice for 2 and 4 weeks stained using Von Kossa. The intensity of the dark brown-black dye of von Kossa implies calcium deposition in the sponges [24]. The part that can be seen in black is the part where the mineral is deposited (HA) in the scaffolds, and the bone cell is exist around it. Compared with the DC/ HAp sponges, the EGCG/DC/HAp sponges displayed higher staining intensity and calcium deposition. Noteworthy, we observed a highest Von Kossa staining intensity for the $5 \mu \mathrm{M}$ EGCG/DC/HAp sponges, consistent with the H\&E staining results. The proportion of
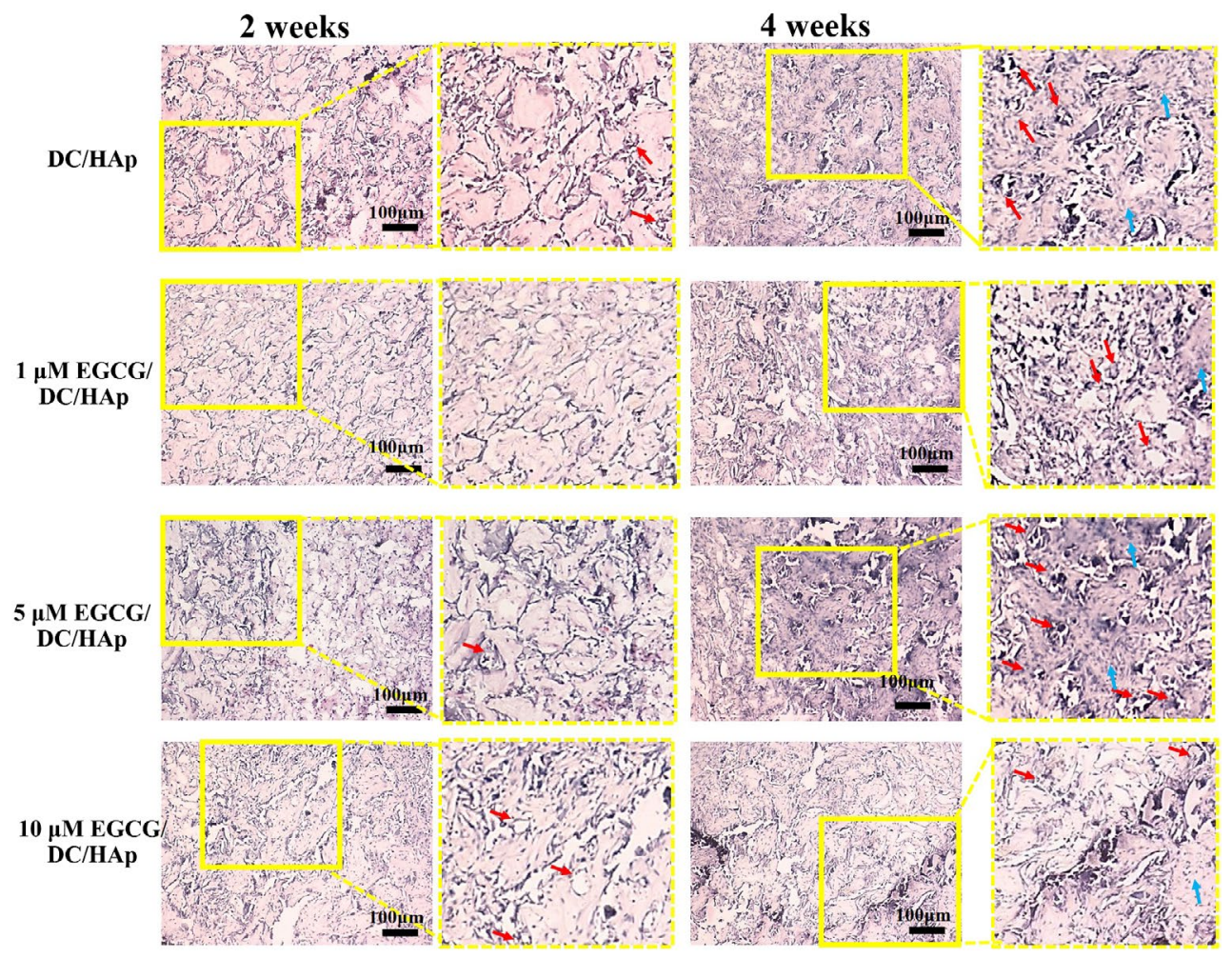

Figure 6. Histological evaluation of EGCG/DC/HAp sponges implanted under the subcutaneous region of athymic nude mice for 2 and 4 weeks stained hematoxylin and eosin $(\mathrm{H} \& \mathrm{E})(\times 40$ maginification, $n=3$ in each group, $p^{*}<0.05$ ) (Please see the online article for the colour version of this figure: https://doi.org/ 10.1080/09205063.2017.1414480).

Note: Red arrow - bone mineralized nodule; blue arrow - osteoblast. 


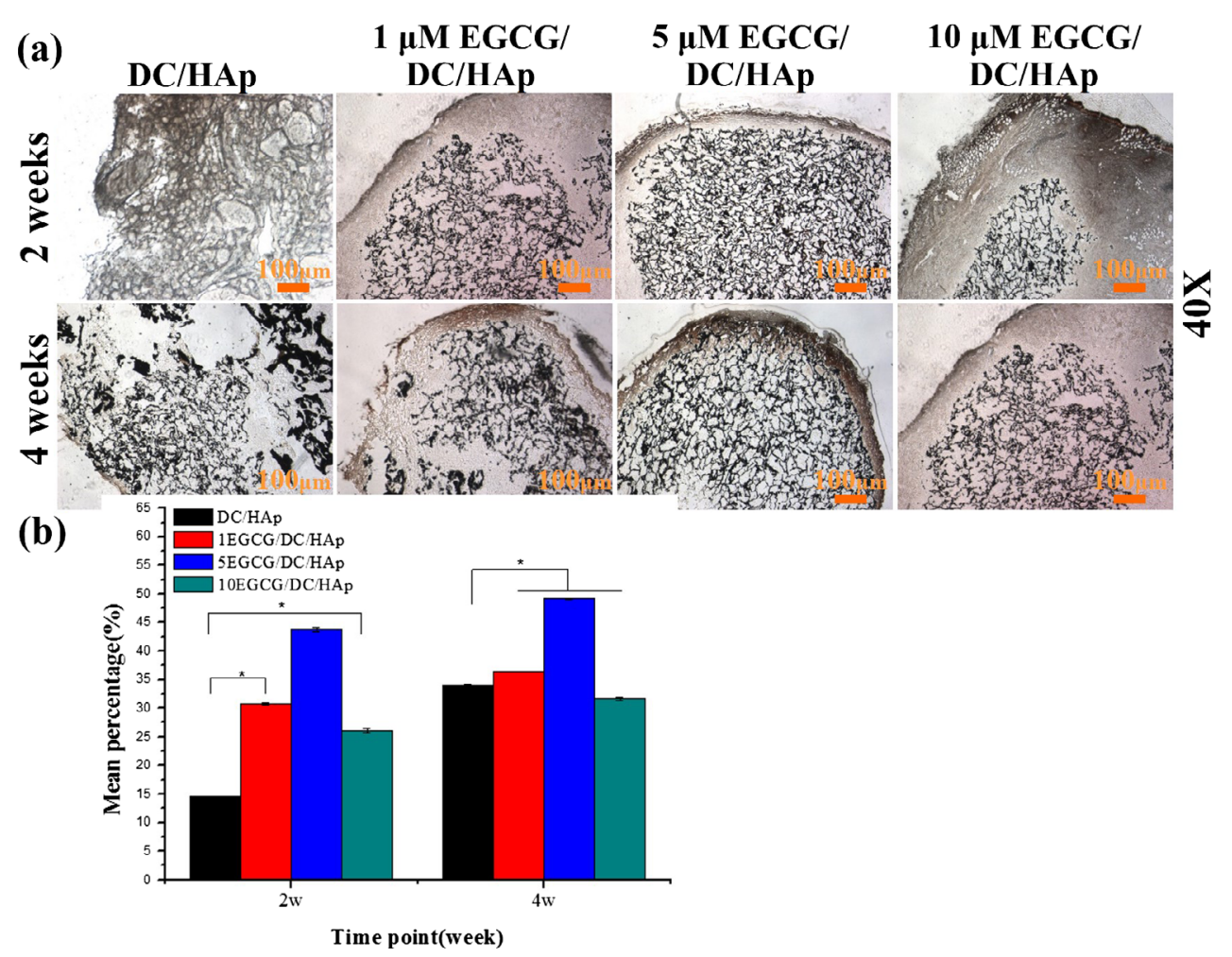

Figure 7. (a) Von Kossa stained section of EGCG/DC/HAp sponges ( $\times 100)$. (b) Evaluation of Von Kossa staining demonstrating calcium deposition within EGCG/DC/HAp sponges $(n=2)$.

calcium deposition to area was plotted by the Image J program Figure 7(b). When comparing the mean percentage of Von Kossa values of 2 and 4 weeks, DC/HAp is $14.585 \pm 0.023$, $33.984 \pm 0.132,1 \mu \mathrm{M}$ EGCG/DC/HAp is $30.799 \pm 0.1423,33.984 \pm 0.132,5 \mu \mathrm{M}$ EGCG/ DC/HAp is $43.817 \pm 0.4332,49.159 \pm 0.1323$ and $10 \mu \mathrm{M}$ EGCG/DC/HAp is $26.048 \pm 0.412$, $31.64 \pm 0.313$ respectively. EGCG at concentrations of 1 and $5 \mu \mathrm{M}$ caused a dose-dependent increase in the number and area of mineralized bone nodules as assessed by Von Kossa. But $10 \mu \mathrm{M}$ is lower value comparing 1 and $5 \mu \mathrm{M}$ EGCG/DC/Hap. This result that excessive addition of EGCG was found to down regulates osteoblast maturation by inhibiting latestage differentiation [25].

\section{Conclusions}

We have fabricated DC/HAp, 1, 5, and $10 \mu \mathrm{M}$ EGCG/DC/HAp sponges to evaluate its efficiency as potential scaffold for healing bone defects and stimulating bone regeneration. The sponges displayed an increased mechanical strength owing to more compact, dense pores and high compressive strength as added EGCG to the DC/HAp sponges. The EGCG/ DC/HAp sponges also demonstrated improved cell viability and osteoinduction, since the presence of EGCG in the sponges have significantly up-regulated expression of osteogenic genes and the formation of bone-like nodules. Further exploration has indicated that EGCG directs osteogenic differentiation via the continuous up-regulation of Runx2, OCN, COL 
1 on 1 and $5 \mu \mathrm{M}$ EGCG/DC/HAp. We identified by H\&E and Von Kossa staining. H\&E staining results show well-defined construct and cell distribution in all experiment groups. As a result of the $H \& E$ staining, after 2 weeks, there was space but after 4 weeks It was confirmed that there was osteoblast in the space of the empty sponge and we indicate osteoblast. In particular, the $5 \mu \mathrm{M}$ EGCG/DC/HAp sponges significantly increased bone mineralized nodules. Through Von Kossa staining, the calcium deposition part that can be seen in dark brown is the part where the mineral is deposited (HAp) in the scaffolds, and the bone cell is exist around it.

And particularly, $5 \mu \mathrm{M}$ EGCG/DC/HAp sponge is more brownish and has a larger dark brown area than the other three groups. This suggests that the osteoblast are more present in the $5 \mu \mathrm{M}$ EGCG/DC/HAp sponge. Collectedly, the EGCG/DC/HAp sponge with good physical properties, enhanced cell proliferation may promote the osteogenic differentiation of rBMSCs and act as a chemoattractant to drive bone regeneration. And it could be applied to the human body substitute as a natural material for bone regeneration.

\section{Disclosure statement}

The authors declare no competing financial interest.

\section{Funding}

This research was supported by Technology Commercialization Support Program [grant number 814005-03-3-HD020], MIFAFF; and Basic Science Research Program [grant number NRF2017R1A2B3010270] through the National Research Foundation of Korea, Ministry of Science, ICT and Future Planning, Republic of Korea.

\section{References}

[1] Fishero BA, Kohli N, Das A, et al. Current concepts of bone tissue engineering for craniofacial bone defect repair. Craniomaxillofac Trauma Reconstr. 2015;8:23-30.

[2] Azami M, Moosavifar MJ, Baheiraei N, et al. Preparation of a biomimetic nanocomposite scaffold for bone tissue engineering via mineralization of gelatin hydrogel and study of mineral transformation in simulated body fluid. J Biomed Mater Res A. 2012;100:1347-1355.

[3] Cho CH, Lee SB. Biodegradable collagen matrix (Ologen) implant and conjunctival autograft for scleral necrosis after pterygium excision: two case reports. BMC Ophthalmol. 2016;15;140.

[4] Bitar KN, Zakhem E. Design strategies of biodegradable scaffolds for tissue regeneration. Biomed Eng Comput Biol. 2016;6:13-20.

[5] Kook YJ, Lee DH, Song JE, et al. Osteogenesis evaluation of duck's feet-derived collagen/ hydroxyapatite sponges immersed in dexamethasone. Biomater Res. 2017;21:2.

[6] Kim SH, Park HS, Lee OJ. Fabrication of duck's feet collagen-silk hybrid biomaterial for tissue engineering. Int J Biol Macromol. 2016;85:442-450.

[7] Pawlaczyk I, Czerchawski L, Kanska J, et al. An acidic glycoconjugate from Lythrum salicaria L. with controversial effects on haemostasis. J Ethnopharmacol. 2010;131:63-69.

[8] Quinlan E, Lopez-Noriega A, Thompson E, et al. Development of collagen-hydroxyapatite scaffolds incorporating PLGA and alginate microparticles for the controlled delivery of rhBMP-2 for bone tissue engineering. J Control Release. 2015;198:71-79.

[9] Peter B, Bosze S, Horvath R. Biophysical characteristics of proteins and living cells exposed to the green tea polyphenol epigallocatechin-3-gallate (EGCg): review of recent advances from molecular mechanisms to nanomedicine and clinical trials. Eur Biophys J. 2017;46:1-24. 
[10] Wang WL, Sheu SY, Chen YS, et al. Enhanced bone tissue regeneration by porous gelatin composites loaded with the Chinese herbal decoction Danggui Buxue Tang. PLoS One. 2017;10:e131999.

[11] Ahmed S, Wang N, Lalonde M, et al. Green tea polyphenol epigallocatechin-3-gallate (EGCG) differentially inhibits interleukin-1 beta-induced expression of matrix metalloproteinase- 1 and -13 in human chondrocytes. J Pharmacol Exp Ther. 2004;308:767-773.

[12] Adikesavan G, Vinayagam MM, Abdulrahman LA, et al. (-)-Epigallocatechin-gallate (EGCG) stabilize the mitochondrial enzymes and inhibits the apoptosis in cigarette smoke-induced myocardial dysfunction in rats. Mol Biol Rep. 2013;40:6533-6545.

[13] Albrecht DS, Clubbs EA, Ferruzzi M, et al. Epigallocatechin-3-gallate (EGCG) inhibits PC-3 prostate cancer cell proliferation via MEK-independent ERK1/2 activation. Chem Biol Interact. 2008;171:89-95.

[14] Cai j, Jing D, Shi M, et al. Epigallocatechin gallate (EGCG) attenuates infrasound-induced neuronal impairment by inhibiting microglia-mediated inflammation, J Nutr Biochem. 2014;25;716-725.

[15] Mah YJ, Song JS, Kim SO, et al. The effect of epigallocatechin-3-gallate (EGCG) on human alveolar bone cells both in vitro and in vivo. Arch Oral Biol. 2014;59:539-549.

[16] Jin P, Wu H, Xu G, et al. Epigallocatechin-3-gallate (EGCG) as a pro-osteogenic agent to enhance osteogenic differentiation of mesenchymal stem cells from human bone marrow: an in vitro study. Cell Tissue Res. 2014;356:381-390.

[17] Song JE, Lee SE, Cha SR, et al. Inflammatory response study of gellan gum impregnated duck's feet derived collagen sponges. J Biomater Sci Polym Ed. 2016;27:1495-1506.

[18] de Almeida OM, Jorgetti W, Oksman D, et al. Comparative study and histomorphometric analysis of bone allografts lyophilized and sterilized by autoclaving, gamma irradiation and ethylene oxide in rats. Acta Cir Bras. 2013;28:66-71.

[19] Boskey A, Pleshko Camacho N. FT-IR imaging of native and tissue-engineered bone and cartilage. Biomaterials. 2007;28:2465-2478.

[20] Pati F, Song TH, Rijal G. Ornamenting 3D printed scaffolds with cell-laid extracellular matrix for bone tissue regeneration. Biomaterials. 2015;37:230-241.

[21] Qin Y, Wang L, Gao Z. Bone marrow stromal/stem cell-derived extracellular vesicles regulate osteoblast activity and differentiation in vitro and promote bone regeneration in vivo. Sci Rep. 2016;6:21961.

[22] Boonrungsiman S, Gentleman E, Carzaniga R, et al. The role of intracellular calcium phosphate in osteoblast-mediated bone apatite formation. Proc Natl Acad Sci USA. 2012;109;14170-14175.

[23] Tominari T, Matsumoto C, Watanabe K, et al. Epigallocatechin gallate (EGCG) suppresses lipopolysaccharide-induced inflammatory bone resorption, and protects against alveolar bone loss in mice. FEBS Open Bio. 2015;5:522-527.

[24] Jeon OH, Panicker LM, Lu Q. Human iPSC-derived osteoblasts and osteoclasts together promote bone regeneration in 3D biomaterials. Sci Rep. 2016;6:1-11.

[25] Vali B, Rao LG, El-Sohemy A. Epigallocatechin-3-gallate increases the formation of mineralized bone nodules by human osteoblast-like cells. J Nutr Biochem. 2007;18(5):341-347. 